\title{
ESTUDO ULTRASTRUTURAL DE BIOPSIAS DE CONJUNTIVA EM DOENÇAS METABÓLICAS DO SISTEMA NERVOSO
}

\author{
S. ROSEMBERG * \\ C. CAMPOS **
}

As doenças metabólicas do sistema nervoso central (SNC), bem que relativamente raras se comparadas com a totalidade dos outros processos patológicos, constituen contingente não negligenciável de alteraçōes responsáveis pela procura de atendimento especializado em serviços de Neurologia Pediátrica. A tentativa de um diagnóstico preciso é extremamente importante para fins prognósticos, de aconselhamento genético e eventualmente terapêutico. Diante de criança suspeita de sofrer de uma afeç̧ão metabólica do sistema nervoso, isto é, que padeça de doença hereditária, progressiva e cuja maior expressão clínica é neurológica, deve-se proceder a uma bateria de exames complementares cujos resultados deverão permitir a máxima aproximação possivel do diagnóstico real. Como, por definição, estas doenças são causadas por anomalias bioquímicas, sua detecção é o alvo final e a garantia do diagnóstico correto. Entretanto, em várias doenças cuja natureza metabólica é certa, a alteração molecular permanece desconhecida, inexistindo a possibilidade de um diagnóstico bioquimico. Algumas destas doenças são consideradas como "degenerativas". O caráter efêmero desta classificação é evidente na medida em que uma simples descoberta bioquímica pode transformar uma doença degenerativa em metabólica ${ }^{1}$. Por outro lado, os procedimentos laboratoriais para a pesquisa de certas alterações bioquimicas, sobretudo concernentes a dosagens da atividade de enzimas lisosomiais, são complexos e caros, achando-se nosso país numa posição pouco privilegiada a este respeito. Assim, a pesquisa de alteraçóes morfológicas permanece um método diagnóstico valioso. Se até há alguns anos a tentação da biópsia cerebral era grande, refreada em parte pelos aspectos éticos envolvidos, a demonstração de que certas alterações celulares em nivel ultrastrutural existem em células fora do SNC, veio em muito facilitar o diagnóstico i,i;. Assim,

Trabalho realizado no Setor de Neuropediatria do Departamento de Pediatria da Faculdade de Ciências Médicas da Santa Casa de Săo Paulo (FCM SC SP) e na Disciplina de Neuropatologia do Departamento de Patologia da Faculdade de Medicina da Universidade de São Paulo (FMUSP): * Professor Pleno do Departamento de Pediatria da FCM SC SP, chefe do Setor de Neuropediatria e Professor Adjunto do Departamento de Patologia da FMUSP, chefe da Disciplina de Neuropatologia; ** Instrutor do Departamento de Pediatria da FCM SC SP. Agradecimento - Aos Drs. Roberto E. Endo e Teruo Alhara do Departamento de Oftalmologia do Hospital da Santa Casa de São Paulo, pela execução das biópsias conjuntivais. 
fragmentos de nervo periférico, pele e conjuntiva ocular 4 podem ser rotineiramente retirados e estudados do ponto de vista ultrastrutural, em crianças suspeitas de certas doenças metabólicas. A gama de afecções passiveis de levar a alteraçōes celulares ultrastruturais fora do SNC é grande, estando nelas incluídas todas as lisosomopatias, as lipofuscinoses ceróides, e, mais recentemente, as doenças peroxissomiais 7 . Certas afeç̧ões "avulsas" como a doença de Lafora e a distrofia neuroaxonal infantil ${ }^{10}$ podem ser responsáveis por comprometimento de células fora do SNC.

No Setor de Neuropediatria do Departamento de Pediatria da Santa Casa de São Paulo, a biópsia de conjuntiva faz parte da rotina da bateria de exames a que é submetida toda criança suspeita de uma doença metabólica. A biópsia de conjuntiva foi eletivamente escolhida pela facilidade de sua execuçāo (não há necessidade de anestesia), pelo seu caráter não invasivo, ocorrendo reparaçāo rápida, sem cicatriz. Este trabalho tem por objetivo relatar nossa experiência com o exame ultrastrutural da conjuntiva ocular no diagnóstico das doenças metabólicas do sistema nervoso.

\section{MATERIAL E METODOS}

O material consiste em 40 biópsias de conjuntiva ocular realizadas em 40 pacientes seguidos no setor de Neuropediatria do Hospital da Santa Casa de Sāo Paulo, no período de 1982 a 1985 . Os critérios clínicos para suspeita de doença metabólica foram previamente determinados $(1,9)$. Dezesseis crianças eram do sexo feminino e 24 do sexo masculino. Dezesseis casos iniciaram sua doença no período infantil precoce $(0$ a 12 meses), 19 no periodo infantil tardio ( 1 a 4 anos) e 5 no período juvenil (acima de 4 anos). A tabela 1 mostra os principais exames laboratoriais realizados nos 40

\begin{tabular}{lccc}
\hline Exame & No pacientes & $\begin{array}{c}\text { Resultado } \\
\text { normal }\end{array}$ & $\begin{array}{c}\text { Resultado } \\
\text { anormal }\end{array}$ \\
\hline RX esqueleto & 21 & 10 & 11 \\
Mielograma & 37 & 25 & 12 \\
LCR & 32 & 30 & 2 \\
Exame ocular & 40 & 25 & 15 \\
Tomografia computadorizada & & & 16 \\
do crânio & 27 & 11 & 9 \\
Dosagem de enzimas & 22 & 13 & \\
lissosomiais: lágrimas e urina & & & \\
\hline
\end{tabular}

Tabela $t$ - Principais exames laboratoriais realizados.

pacientes. Logo após a retirada, o fragmento de conjuntiva é fixado em glutaraldeido a 2\% por 2 horas e ácido ósmico a $1 \%$ por mais duas horas. Após passagem em uranila e desidratação, faz-se inclusăo em araldite, sendo os cortes semi-finos corados pelo azul de metileno. Após escolha das areas representativas, procede-se aos cortes finos que são corados pela uranila e citrato de chumbo. A observaçăo foi feita em um microscópio Zeiss M-952. 


\section{RESULTADOS}

Baseados nos achados das biópsias de conjuntiva ocular, os pacientes foram divididos em dois grandes grupos: Grupo A - biópsias com alteraçōes ultrastruturais: 20 pacientes; Grupo B - biópsias sem alteraçōes ultrastruturais: 20 pacientes. A distribuição das patologias no interior de cada grupo está nas tabelas 2 e 3.

\begin{tabular}{lc}
\hline Diagnóstico & Ne pacientes \\
\hline \hline Mucopolissacaridoses & 8 \\
Gangliosidose $\mathrm{G}_{\mathrm{M} 1}$ & 6 \\
Distrofia neuro axonal infantil & 4 \\
Gangliosidose $\mathrm{G}_{\mathrm{M} 2}$ - Variante $\mathrm{AB}$ & 1 \\
Lipofuscinose ceróide & 1 \\
\hline
\end{tabular}

Tabela 2 - Diagnóstico dos pacientes com biópsias anormais.

\begin{tabular}{lc}
\hline Diagnóstico & No pacientes \\
\hline \hline Doença de Leigh & 1 \\
Doença de Hallervorden-Spatz & 1 \\
Adrenoleucodistrofia & 1 \\
Leucodistrofia metacromática & 1 \\
Sem diagnóstico & 16 \\
\hline
\end{tabular}

Tabela 3 - Diagnóstico dos pacientes com biopsias normais.

\section{COMENTARIOS}

As doenças lisosomiais inatas são as que mais conspicuamente levam a alteraçōes ultrastruturais em células fora do SNC. Os lisosomos ingurgitados com as substâncias não metabolizadas em virtude da baixa atividade enzimática são geralmente bem visualizados. Sua abundância varia segundo a doença. Nas mucopolissacaridoses, sua visualização é imediata: são grandes lisosomos transformados em vacúolos claros contendo no seu interior material filamentar. Estas alterações são intensas, abundantes e presentes em diferentes tipos celulares como células endoteliais, glandulares, fibroblásticas, células de Schwann (Fig. 1). Sendo este aspecto idêntico nos diferentes tipos de mucopolissacaridose, a análise ultrastrutural permite apenas o diagnóstico genérico. As dosagens enzimáticas realizadas em dois casos mostraram tratar-se da MPS III-B (Sanfilippo tipo B) e de MPS I-S (Scheie). Nos demais casos, não se realizaram estas dosagens, porém os quadros clínicos sugeriam as MPS I-H (Hurler) e MPS-II (Hunter). $\mathrm{Na}$ gangliosidose $\mathrm{G}_{\mathrm{M} 1}$, todos pertinentes à forma precoce (doença de Landing), 


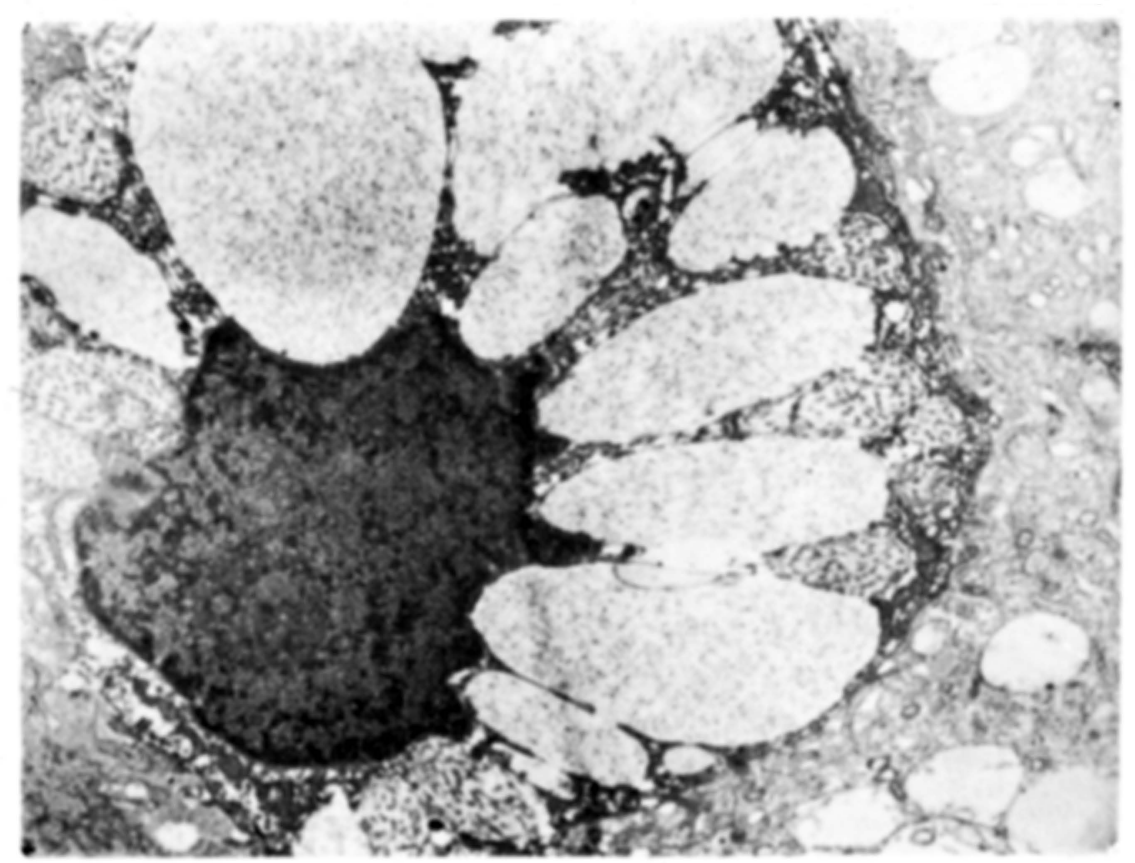

Fig. 1 - Mucopolissacaridose. Células glandulares de conjuntiva cujo citoplasma está inteiramente tomado por vacúolos claros contendo em seu interior material filamentar. $X$.9990.

o aspecto foi o mesmo e o diagnóstico também é facilmente realizado. $O$ aspecto é semelhante ao das mucopolissacaridoses, estando as células epiteliais, endoteliais e fibroblásticas repletas de grandes inclusões citoplasmáticas eletroluscentes, delimitadas por membranas únicas, vazias ou contendo em seu interior escasso material filamentar (Fig. 2). Este aspecto explica-se pelo fato de na gangliosidose $\mathrm{G}_{\mathrm{M}_{1}}$ haver dupla tesaurismose, em gangliosídeos nos neurônios e em mucopolissacárides nas vísceras ${ }^{2}$. Em certos casos, sobretudo no início da evolução, o diagnóstico diferencial entre esta doença e a de Niemann-Pick pode ser clinicamente difícil. A biópsia de conjuntiva mostra padrões completamente diferentes em ambas as entidades, constituindo-se assim em valioso meio diagnóstico. As atterações nas gangliosidoses $\mathrm{G}_{\mathrm{M} 2}$ assim como nas lipofuscinoses ceróides $s$ são muito menos abundantes e requerem busca criteriosa. A presença, na gangliosidose $\mathrm{G}_{\mathrm{M} 2}$, de corpúsculos membrano-citoplasmáticos e de corpos "zebrados" (Fig. 3) é caracteristica, bem que não patognomônica. Em nosso caso, a análise enzimática revelou tratar-se da rara variante A-B da gangliosidose $\mathrm{G}_{\mathbf{M} 2}$. Nas lipofuscinoses ceróides, a presença de grânulos osmiófilos de corpos curvilíneos ou de inclusões tipo “impressão digital” estarão respectivamente predominantes nas formas infantil, infantil tardia e juvenil. Em nosso caso (forma infantil tardia) observamos os corpos curvilíneos em fibroblas- 


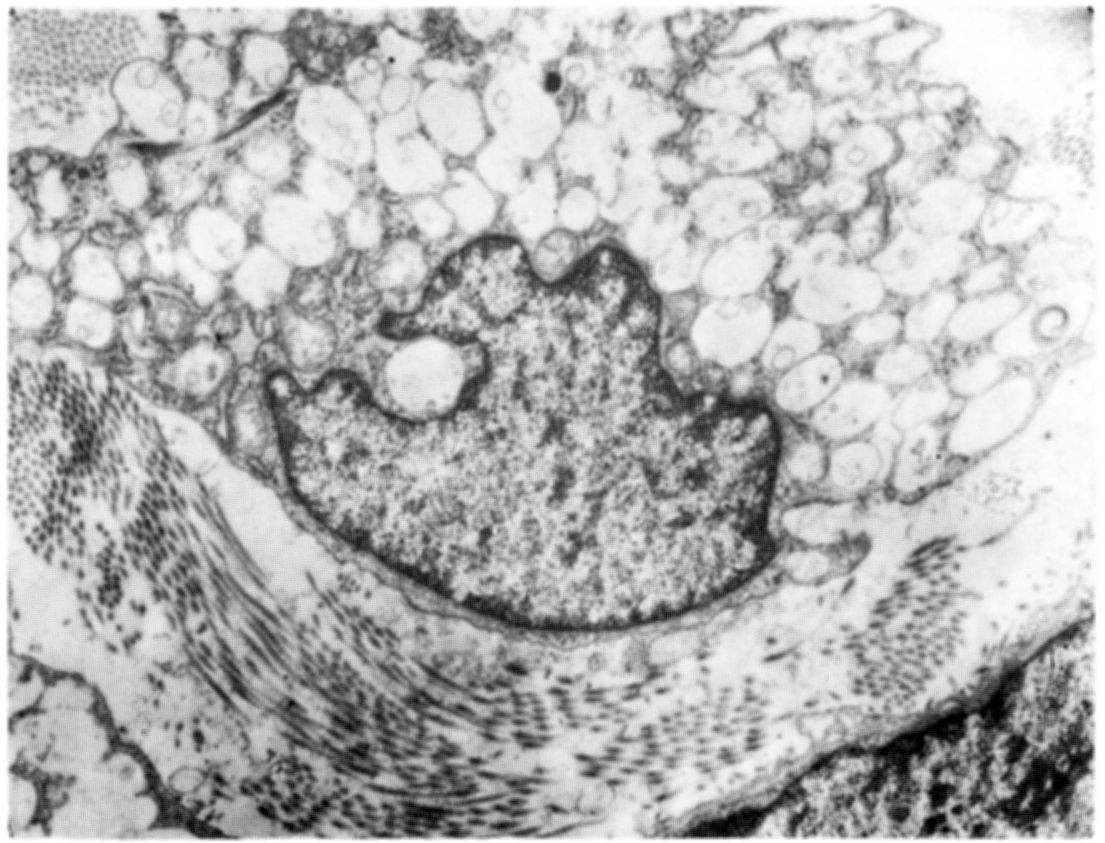

Fig. 2 - Gangliosidose $G_{M i}$. Fibroblasto cujo citoplasma esta preenchido por vacúolos claros. $X 11270$.

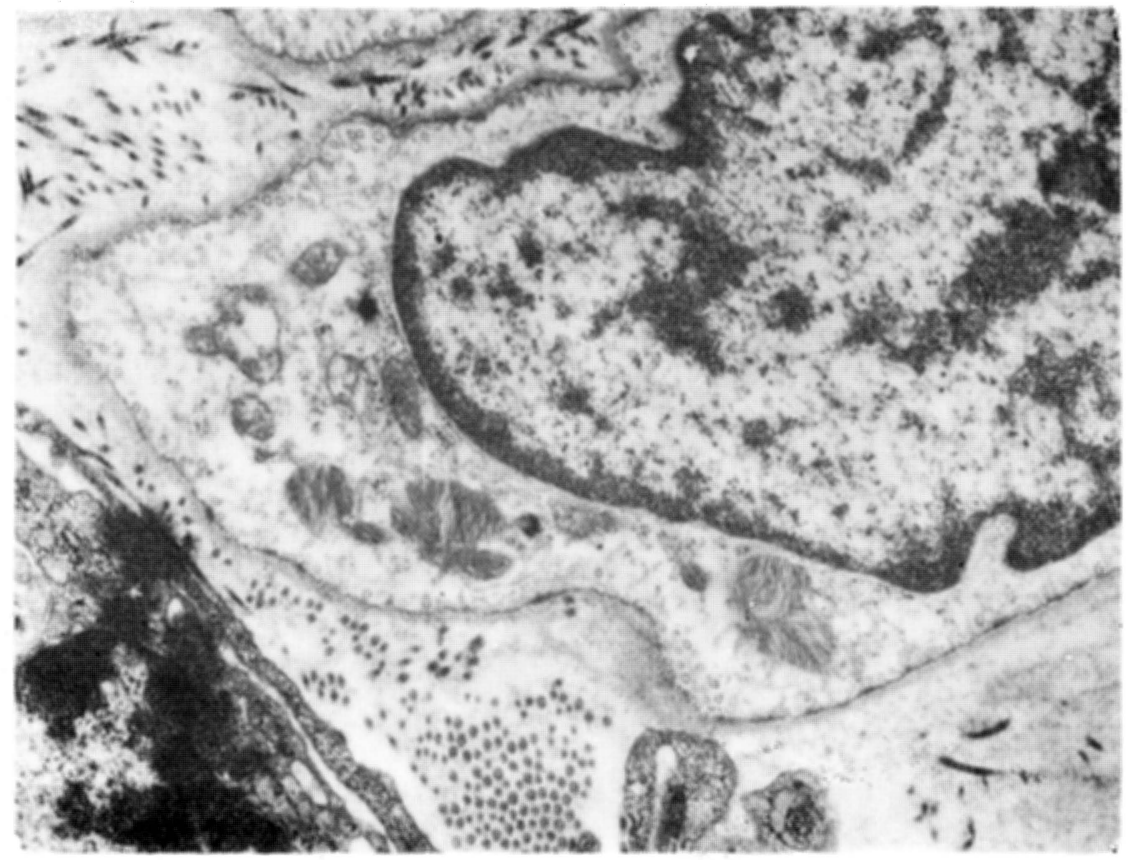

Fig. 3 - Gangliosidose $G_{M 2}$ (Variante $A-B$ ). Célula pericitaría em cujo citoplasma encontram-se numerosas inclusões limitadas por membrana única, constituida de feixes de membranas paralelas. $X 16170$. 


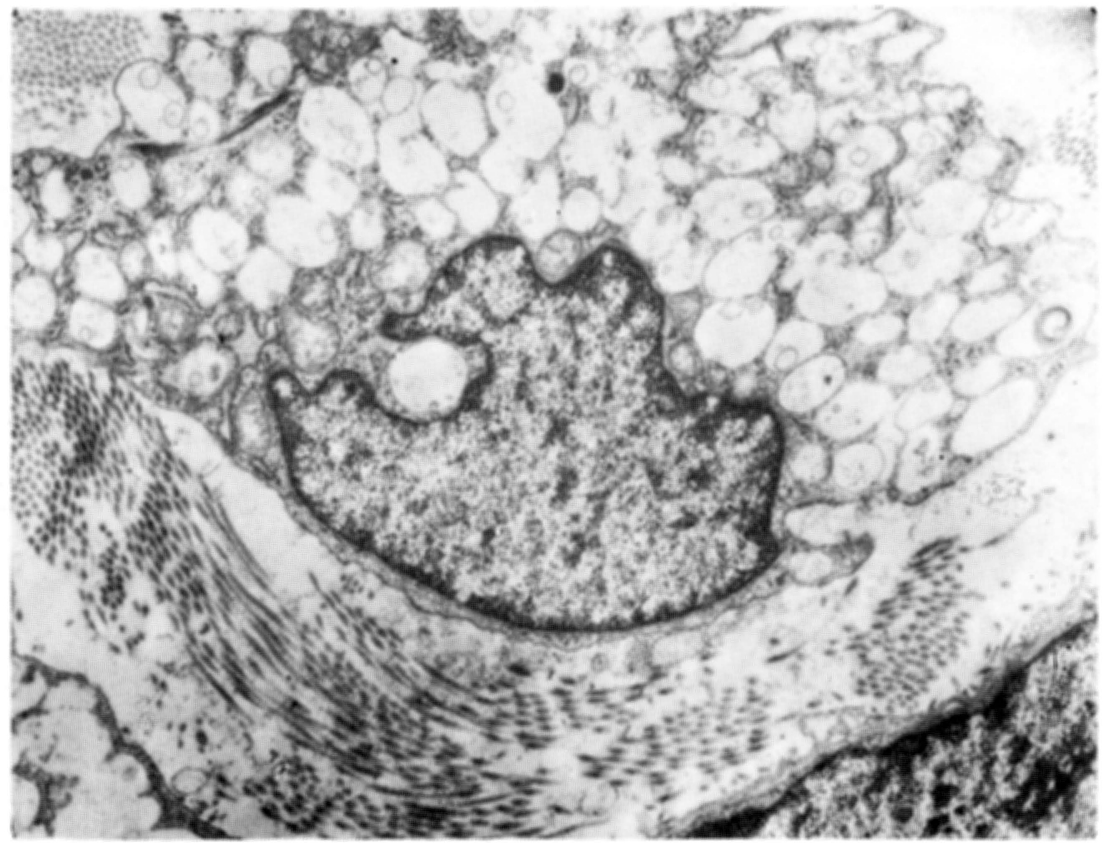

Fig. 2 - Gangliosidose $G_{M i}$. Fibroblasto cujo citoplasma está preenchido por vacúolos claros. $X 112 \% 0$.

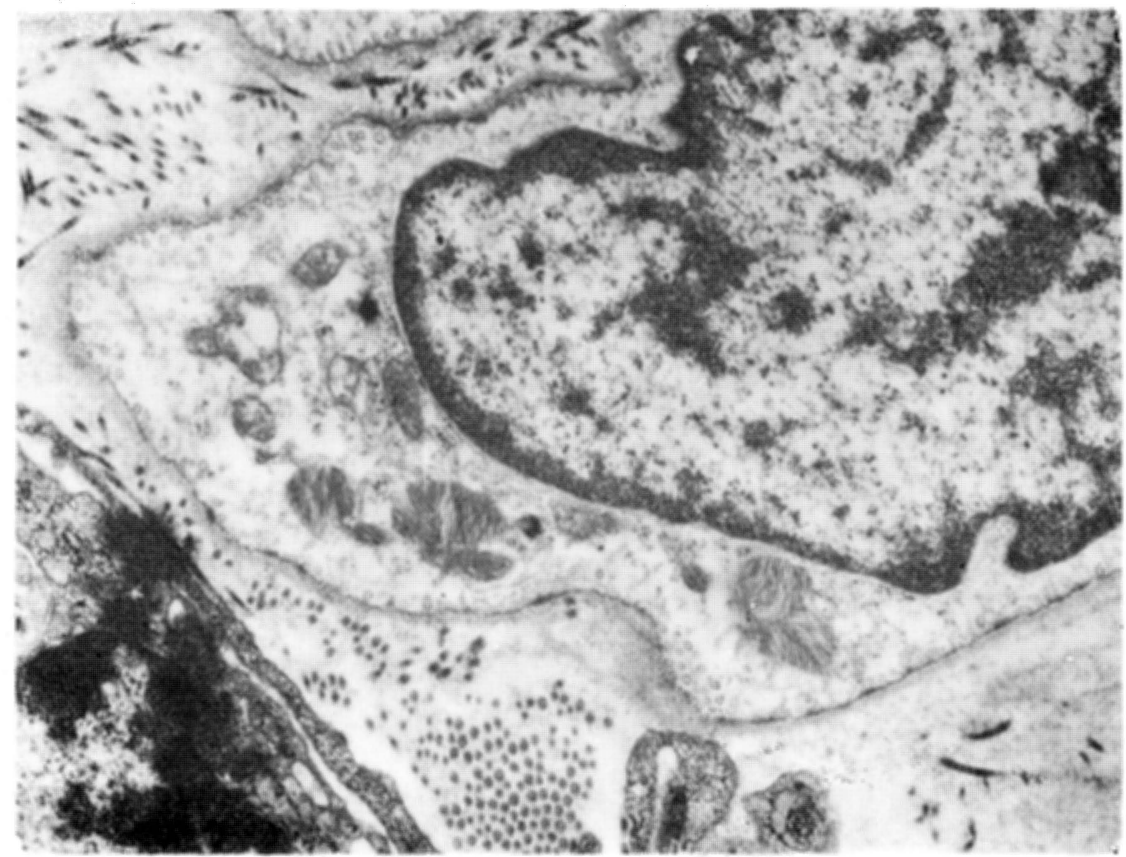

Fig. 3 - Gangliosidose $G_{H_{2}}$ (Variante $A-B$ ). Célula pericitária em cujo citoplasma encontram-se numerosas inclusões limitadas por membrana única, constituída de feixes de membranas paralelas. X 16170. 
tos (Fig. 4) e em células de Schwann. A distrofia neuro-axonal infantil carece de qualquer especificidade bioquímica, sendo seu diagnóstico unicamente possível mediante o achado de neurônios distróficos ${ }^{10}$. Estes são visiveis principalmente nos nervos amielínicos conjuntivais (Fig. 5).

Em vinte casos, a biópsia da conjuntiva revelou-se normal. Destes, em dois casos, a negatividade da biópsia foi compreensivel na medida em que os diagnósticos de doença de Leigh e de Hallervorden-Spatz foram confirmados a posteriori através de autópsia e de outros exames paraclinicos, respectivamente. Em 4 casos de sindromes juvenis extrapiramidais progressivas e em dois casos familiais de uma doença progressiva ligada ao sexo, a biópsia negativa permitiu afastar, nestes quadros clinicos não conformes, a possivel natureza lisosomial das afecções. Outros seis casos dizem respeito a observaçōes de pacientes dificilmente agrupáveis por suas características clínicas, nas quais a natureza degenerativa da afecção parecia evidente. Embora sua evolução e quadro clínico nāo sugeriam, na maioria das vezes, uma doença de acúmulo, a biópsia conjuntival foi realizada em funçāo das próprias dificuldades diagnósticas e pelo fato de a literatura conter, cada vez em maior número, casos tidos como variantes atípicas de doenças bem conhecidas ${ }^{1}$. Na observaçăo em que a dosagen da atividade enzimática foi realizada nas lágrimas e urina, esta revelou-se normal. Quatro casos eram clinicamente sugestivos de uma lipofuscinose ceróide, se bem que os cxames complementares (mielograma, EEG) não corroborassem esta hipótese. A negatividade da biópsia deveria em principio afastá-la definitivamente. Atualmente, porém, com maior experiência, sabe-se que as inclusōes nestas afecções não são muito freqüentes nem ubiquitárias. E possivel que dentre estes casos, laaja alguns falsos negativos, fato não confirmado pela impossibilidade do diagnóstico definitivo, uma vez que estes pacientes nāo puderam ser seguidos.

Apenas dois casos foram considerados como verdadeiros falsos negativos: uma leucodistrofia metacromática (diagnosticada através de dosagem enzimática e biópsia de nervo periférico) e uma adrenoleucodistrofia. Nestas duas entidades, a biópsia conjuntival não é a melhor-escolha, justamente pela possibilidade de ai nāo ser o local de mais fácil visualização das inclusōes citoplasmáticas, preferindo-se nervo periférico e testiculo, respectivamente.

Nossos achados indicam que, efetivamente o estudo ultrastrutural da biópsia de conjuntiva é por si mesmo, ou associadamente a outros exames laboratoriais, um instrumento decisivo para o diagnóstico das doenças metabólicas primárias do SNC, mormente quando os complexos técnicos bioquimicos não se acham ao alcance. 


\section{RESUMO}

Quarenta biópsias de conjuntivas obtidas de um numero igual de crianças portadoras de doenças metabólicas do SNC, ou supostas de tal acometimento, foram analisadas do ponto de vista ultrastrutural. Em 20 casos foram observadas alteraçōes ultrastruturais que possibilitaram firmar o diagnóstico de mucopolissacaridose em 8 , gangliosidose $\mathrm{G}_{\mathrm{Mx}}$ em 6 , distrofia neuro-axonal infantil em 4, gangliosidose $\mathrm{G}_{M_{2}}$ em um e forma infantil tardia de lipofuscinose ceróide em um. Nos 20 casos em que o exame revelou-se negativo, este era esperado em dois (doença de Lcigh e de Hallervorden-Spatz); em dois, o resultado revelou-se falso negativo (leucodistrofia metacromática e adrenoleucodistrofia); em 12 casos sua negatividade permitiu afastar doença de acúmulo; em 4 casos suspeitos de lipofuscinose ceróide seu resultado foi inconclusivo. O estudo ultrastrutural de biópsias de conjuntiva é um instrumento importante para o diagnóstico de doenças metabólicas do SNC.

\section{SUMMARY}

Ultrastructural study of conjunctival biopsies in metabolic diseases of the nervous system.

Forty conjunctival biopsies from children suffering from metabolic diseases of the CNS were studied ultrastructurally. In 20 cases they were abnormal ( 8 mucopolysaccharidosis, $6 \mathrm{G}_{\mathrm{M} 1}$ gangliosidosis, 4 infantile neuroaxonal dystrophy, $1 \mathrm{G}_{\mathrm{ML}}$ gangliosidosis, 1 late infantile ceroid lipofuscinosis). In the 20 remaining cases the biopsies did not show abnormalities. From these, 2 were proven as Leigh disease and Hallervorden-Spatz disease in which there is no conjunctival ultrastructural alterations; in 2 cases (one metachromatic leukodystrophy and one adrenoleukodystrophy) the results were false negative); in 4 clinicaly suspected cases of late infantile ceroid lipofuscinosis no alterations were observed and the results were not conclusive. In the remaining 12 cases the negatives allowed to rule out lysosomal disorders. The ultrastructural study of the conjunctival biopsy is an important tool for the diagnosis of neurological metabolic diseases of children mainly when sophisticated biochemical procedures are not available.

\section{REFERANCIAS}

1. ADAMS, R.D. \& LYON, G. - Neurology of Hereditary Metabolic Diseases of Children. Hemisphere Publish. Corporation, Washington, 1982.

2. ARITA, F.N.; CAMPOS, C.C. \& ROsEMBERG, S. - Gangliosidose G 1 forma precoce. Aspectos clínicos e diagnósticos. (A propósito de quatro observações). Pediatria 58:299, 1985 .

3. ARSENIO-NUNES, M.L. \& GOUTIERES, G. - An ultra-microscopic study of the skin in the diagnosis of the infantile and late infantile types of ceroid-lipofuscinosis. J. Neurol. Neurosurg. Psychiat. 38:994, 1975.

4. ARSENIO-NUNES, M.L.; GOUTIERES, F. \& AICARDI, J. - An ultramicroscopic study of skin and conjunctival biopsies in chronic neurological disorders of childhood. Ann. Neurol, 9:163, 1981. 
5. CARPENTER, S.; KARPATTI, G. \& ANDERMANN, F. - Specific involvement of muscle, nerve and skin in late infantile and juvenile amaurotic idiocy. Neurology $22: 170,1972$.

6. CEUTERICK, C.L.; MARTIN, J.J.; CASAER, P. \& EDGAR, G.W. - The diagnosis of infantile generalized ceroid-lipofuscinosis (type Hagberg-Santavuori) using skin biopsy. Neuropediatrie 7:250, 1976.

7. MOSER, H.W. - Peroxisomal disorders. J. Pediat. 108:89, 1986.

8. RAPOLA, J.; SANTAVUORI, P. \& SAVILARTI, E. - Suction biopsy of rectal mucosa in the diagnosis of infantile and juvenile types of neuronal ceroid lipofuscinosis. Hum. Pathol. 15:352, 1984.

9. ROSEMBERG, S. - A criança que involui (encefalopatia evolutiva). In J. Murakovschi - Pediatria. Diagnóstico + Tratamento. Ed. 2. Sarvier, Săo Paulo, 1981, pg. 233.

10. ROSEMBERG, S.; ARITA, F.N. \& CAMPOS, C. -- Distrofia neuroaxonal infantil. Diagnóstico em vida por biópsia conjuntival. Arq. Neuro-Psiquiat. (São Paulo) $43: 48,1985$.

Departamento de Patologia, Faculdade de Medicina, Universidade de Säo Paulo Caixa Postal 2921 - 01000, São Paulo, SP - Brasil. 